\title{
Non-Translation Invariant Gibbs States with Coexisting Phases III: Analyticity Properties *
}

\author{
Jean Bricmont, Joel L. Lebowitz, and Charles E. Pfister ${ }^{\star \star}$ \\ Department of Mathematics, Rutgers University, New Brunswick, NJ 08903, USA
}

\begin{abstract}
We consider the spatially inhomogeneous Gibbs states for the three dimensional Ising and Widom-Rowlinson models. We prove the analyticity in $z=\exp (-2 \beta J)$ for small $|z|$ of the spin correlation functions of these Gibbs states and of the surface tension.
\end{abstract}

\section{Introduction}

We consider the three-dimensional Ising model, with nearest neighbor ferromagnetic interactions, in a box with \pm boundary conditions (b.c.); the spins surrounding the top (bottom) half of the box are equal to $+1(-1)$. It is known $[1,2]$ that, at low temperatures, these b.c. lead, in the thermodynamic limit, to an extremal non-translation invariant Gibbs state whose correlation functions cluster exponentially.

In this paper we prove that these correlation functions are analytic in the variable $z=\exp (-2 \beta J)$ for $|z|$ sufficiently small; $\beta$ is the inverse temperature and $J$ is the coupling. We also study the surface tension $\tau$, i.e., the thermodynamic limit of the difference in free energy per unit cross-section between the system with \pm b.c. and one with + b.c. (all spins on the boundary equal to +1 ). We show that $\tau-2 \beta J$ is analytic in $z$ at low temperatures. Moreover, we extend our results to Widom-Rowlinson models on a lattice; the systems discussed in the first two papers of this series $[3,4]$.

The method we use is a low-temperature expansion of the Minlos-Sinai or Kirkwood-Salzburg type. (This is similar to the method used by Gallavotti in his work [5] on the two-dimensional Ising model with \pm b.c.) The Minlos-Sinai equations [6] were originally developed for studying the low-temperature behavior of the pure phase, i.e., the state obtained with the + b.c. The configurations of the system with + b.c. are described by means of contours and Minlos and Sinai apply Kirkwood-Salzburg equations to the 'gas' of contours. The factor $\exp (-2 \beta J)$ plays the role of an activity.

* Supported in part by N.S.F. Grant PHY 77-22302

$\star \star$ Supported by the Swiss National Foundation for Scientific Research 
In the \pm b.c., we first remark that for each configuration there is an interface, i.e., a contour that is connected to the part of the boundary separating the + and - spins. By definition, this interface divides the box into two regions having pure + or - b.c. Our strategy will be to concentrate on the interface and then use the Minlos-Sinai low-temperature expansion for the pure phases.

Dobrushin has shown that the interface can be decomposed into elementary excitations in the following way: in the ground state, the interface is perfectly flat. At non-zero temperatures, however, the interface will be deformed: these deformations are called walls in [1] and, once specified, completely determine the interface. We do a low-temperature expansion for the correlation functions of these walls. Unlike the contours which interact only through a hard-core, our walls have in addition complicated (effective) interactions. These come about because a modification of the interface also changes the regions with pure + and - b.c. On the other hand, very large walls are unlikely in the system with \pm b.c. for the same reason that long contours are unlikely with + b.c. It turns out that all we need to control the interactions between the walls is good control on the pure phases and this is given by the Minlos-Sinai expansion. We can then apply a more or less standard Kirkwood-Salzburg expansion for the correlation functions of the walls.

Associated with these walls is a free energy that turns out to be related to the surface tension and therefore our expansion shows also the analyticity of the latter.

One may ask: what is the relationship with Gallavotti's work mentioned above? There, the physical situation is completely different, since the same b.c. leads to a translation invariant Gibbs state in two dimensions. However, to show that the separation line (analogue of our interface) fluctuates, Gallavotti uses essentially the same decomposition into walls and then does a low-temperature expansion to show that these walls (called 'jumps') are almost independent. The difference between two and three dimensions is geometrical. It comes from the fact that in two dimensions large deviations of the interface from its flat ground state can be produced by the addition of a large number of small jumps, while in three dimensions this is impossible.

Let us mention that we use the 'algebraic formalism' instead of the equations in Banach spaces to perform our expansions. Although we strongly rely on [5], we assume only a knowledge of the algebraic formalism as expressed, e.g., in [7].

We also recall without proofs the results of Dobrushin [1] on the geometry of the interface.

Since we are interested in quantities whose existence in the thermodynamic limit follows from correlation inequalities for real values of $\beta J$, we simplify matters somewhat by using Vitali's Theorem to prove analyticity of the limit functions. This requires only giving uniform bounds for the quantities under consideration rather than controlling the expansion term by term.

\section{Outline}

In Sect. 2 we state the main results (Theorem 1). Section 3 is devoted to the case of the pure phases. We introduce the contours and give in Theorem 2 (which is 
not proved because it is standard) the main estimates on these. Theorem 3 gives an expansion for the spin correlation functions in the pure phase which is very convenient for our purposes. This is proven in Appendix 1 and is due to Kunz and Souillard [8].

Section 4 deals with the \pm b.c. and the geometry of the interface. We give there the connection between the interactions of the walls and the properties of the pure phases. Also given there is the relation between the spin correlation functions for the \pm b.c. and the correlation functions of the walls. In Theorem 4 we state the necessary estimates on the correlation functions of the walls. This is the analogue of Theorem 2 for the pure phase.

In Sect. 5 we give and prove the basic ingredients for the proof of Theorem 4 , that is the estimates on the potentials (interactions) between the walls. This relies basically on Theorem 2. The actual proof of Theorem 4 is deferred to Appendix 2.

Section 6 gives the proof of Theorem 1, combining Theorem 3 (expansion of the spin correlation functions in the pure phases) and Theorem 4 (estimates on the correlation functions of the walls).

Appendix 3 is devoted to the Widom-Rowlinson models [3,4]. With the correct definition of contours, all our results extend immediately and we do not, therefore, give many details.

\section{Main Results}

Let $i=\left(i_{1}, i_{2}, i_{3}\right) \in \mathbb{Z}^{3}$ specify a lattice site and $\sigma_{1}$ be a spin variable with values \pm 1 . A configuration on $\Lambda \subset \mathbb{Z}^{3}$ is $\sigma_{\Lambda}=\left(\sigma_{i}, i \in \Lambda\right)$. We put $\sigma_{\mathbb{Z}^{3}}=\sigma$. Given $\Lambda \subset \mathbb{Z}^{3}$ we can always write $\sigma=\left(\sigma_{\Lambda}, \sigma_{\Lambda^{c}}\right)$ where $\Lambda^{c}=\mathbb{Z}^{3} \backslash \Lambda$. We denote by $\langle i, j\rangle$ a pair of nearest neighbors sites $i$ and $j$ of $\mathbb{Z}^{3}$. The energy of a configuration $\sigma_{\Lambda}$, given $\sigma_{\Lambda}, \Lambda$ finite, is

$$
H\left(\sigma_{\Lambda} \mid \sigma_{\Lambda^{c}}\right)=-J \sum_{\langle i, j\rangle} \sigma_{i} \sigma_{j}, \quad J>0
$$

where the sum is restricted to all $\langle i, j\rangle$ such that $i \in \Lambda$. We choose now a special set $\Lambda: \Lambda=A_{L, M}=\left\{i \in \mathbb{Z}^{3}:-M \leqq i_{1}<M,-L \leqq i_{2}, i_{3}<L\right\} L$ and $M$ being positive integers. We define three boundary conditions for $A_{L, M}$, the + b.c., the - b.c., and the \pm b.c., by specifying the values of $\sigma_{j}$ for $j \in A_{L, M}^{c}$ as follows

$$
\begin{aligned}
+ \text { or }- \text { b.c. }: \sigma_{j} & =+1 \text { or }-1 \forall j \in A_{L, M}^{c} \\
\pm \text { b.c. }: \sigma_{j} & =\left\{\begin{array}{ll}
+1 & j_{1} \geqq 0 \\
-1 & j_{1}<0
\end{array} \forall j \in A_{L, M}^{c} .\right.
\end{aligned}
$$

Let $\alpha$ be,+- , or \pm . The energy of the configuration $\sigma_{A_{L, M}}$ given the $\alpha$ b.c. is denoted by $H_{L, M}\left(\sigma_{A_{L, M}} \mid \alpha\right)$. The corresponding partition function is

$$
Z_{L, M}^{\alpha}=\sum_{\substack{\sigma_{L}= \pm 1 \\ i \in A_{L, M}}} \exp \left(-\beta H_{L, M}\left(\sigma_{A_{L, M}} \mid \alpha\right)\right)
$$

where $\beta>0$ is the inverse temperature. We denote by $\langle\cdot\rangle_{L, M}^{\alpha}$ the expectation value for the Gibbs state in $A_{r}$. w with $\alpha$ b.c. Let $B$ be a finite subset of $\mathbb{Z}^{3}$. We define 
$\sigma^{B}=\prod_{i \in B} \sigma_{i}$ and the spin correlation functions by $\left\langle\sigma^{B}\right\rangle_{L, M}^{\alpha}$. These functions depend on $\beta J$. At low temperature the \pm b.c. yields, in the thermodynamic limit, a nontranslation invariant Gibbs state in which the two pure phases, obtained for $\alpha=+$ and $\alpha=-$, are separated by an interfacial region. The surface tension $\tau$ between these two phases is defined by [9]

$$
\tau=-\lim _{L \rightarrow \infty} \frac{1}{(2 L)^{2}} \lim _{M \rightarrow \infty} \log \frac{Z_{L, M}^{ \pm}}{Z_{L, M}^{+}} .
$$

Theorem 1. Let $z=e^{-2 \beta J}$. Then there exists $r>0$ such that:

(a) for real $z, 0<z<r, \tau(\beta J)$ exists and $\tau(\beta J)-2 \beta J$ is an analytic function of $z,|z|<r$

(b) for every finite $B \subset \mathbb{Z}^{3}$

$\lim \lim \left\langle\sigma^{B}\right\rangle_{L, M}^{ \pm}=\left\langle\sigma^{B}\right\rangle^{ \pm}$is analytic in $z,|z|<r$. $L \rightarrow \infty M \rightarrow \infty$

Remarks. The existence of the limits in Theorem 1 can be proven with the method of this paper, or by using correlation inequalities $[9,10]$. Actually using correlation inequalities one proves existence for all $\beta J$. The proof of the analyticity of $\left\langle\sigma^{B}\right\rangle^{ \pm}(z)$ is done by showing first that $\lim _{M \rightarrow \infty}\left\langle\sigma^{B}\right\rangle_{L, M}^{ \pm}(z)$ is analytic in $z,|z|<r$ and uniformly bounded in $L$. Then one uses Vitali's Theorem.

\section{The Pure Phase}

We recall now some relevant results concerning the pure phases. Although the analysis below is originally due to Minlos and Sinai [6], most of it can be found in [7]. Some new results (Theorem 3) are due to Kunz and Souillard [8]. We use the algebraic formalism [7].

Let $E$ be a set with elements $x, y$ etc. such that there is a notion of 'being adjacent' between the elements of $E$. Then we say that a subset $F \subset E$ is connected if for any $x$ and $y$ of $F$ we can find a sequence $x_{1}, \ldots, x_{n}$ of elements of $F$ such that $x_{1}=x$, $x_{n}=y, x_{i}$ and $x_{i+1}$ are adjacent, $i=1, \ldots, n-1$. Connected subsets of $\mathbb{R}^{3}$ are defined with the usual topology on $\mathbb{R}^{3}$.

We consider now the lattice $\mathbb{Z}^{3}$. To each pair of nearest neighbor sites, $\langle i, j\rangle$, we associate the unit closed face perpendicular to the segment with endpoints $i$ and $j$ and passing through the middle of the segment. We call an edge any side of a face. Let $\mathscr{H}$ be the set of all these faces. Two faces in $\mathscr{H}$ are adjacent if their union is a connected subset of $\mathbb{R}^{3}$. Given a configuration in $A_{L, M}$ with + b.c. we consider the set of all faces of $\mathscr{H}$ associated to $\langle i, j\rangle$ with $\sigma_{i} \neq \sigma_{j}$. We decompose this subset into maximally connected components called contours. For every contour $\gamma$ of a configuration in $A_{L, M}$ there exists exactly one configuration $\sigma_{\gamma}$ in $A_{L, M}$ which contains only this contour. We write $\gamma \subset A_{L, M}$. We denote by $\mathscr{C}$ the set of all possible contours $\gamma$ in $\mathbb{Z}^{3}$. Two contours $\gamma_{1}$ and $\gamma_{2}$ are compatible if there exists a configuration in some $A_{L, M}$ with + b.c. containing exactly these two contours. We define the interior of $\gamma$, Int $\gamma$, as the subset of all sites of $\mathbb{Z}^{3}$ where $\sigma_{\gamma}$ is equal to -1 and the exterior of $\gamma$, Ext $\gamma$, as the subset of all sites where $\sigma_{\gamma}$ is 
equal to +1 . There is a bijection between configurations in $A_{L, M}$ with + b.c. and families of contours $\left(\gamma_{1}, \ldots, \gamma_{n}\right)$ with $\gamma_{i} \subset A_{L, M}, i=1, \ldots, n, \gamma_{i}$ compatible with $\gamma_{i}, i \neq j, i, j=1, \ldots, n$. Such a family of contours is called admissible. We identify this family with its characteristic function $\Gamma$ on $\mathscr{C}: \Gamma(\gamma)=1$ if $\gamma$ belongs to the family, $\Gamma(\gamma)=0$ otherwise.

To use the algebraic formalism we consider also non-admissible families of contours. These include families in which a contour $\gamma$ occurs several times. These families are identified with the functions $\Gamma: \mathscr{C} \rightarrow \mathrm{N}$ such that $N(\Gamma)=\sum_{\gamma} \Gamma(\gamma)<\infty$. $\Gamma(\gamma)$ is the multiplicity of the contour $\gamma$ in the family. The function identically zero is denoted by 0 . Let $\mathscr{L}$ be the set of all these functions. We define on $\mathscr{L}$ an addition by $\left(\Gamma+\Gamma^{\prime}\right)(\gamma)=\Gamma(\gamma)+\Gamma^{\prime}(\gamma)$. We say that $\Gamma \leqq \Gamma^{\prime}$ if and only if $\Gamma(\gamma) \leqq \Gamma^{\prime}(\gamma) \forall \gamma$. We define the length of a contour $\gamma$ by $|\bar{\gamma}|$, i.e., cardinality of $\gamma \subset \mathscr{H}$, and $|\Gamma|=\sum_{\gamma} \Gamma(\gamma)|\gamma|$. We also use the notation $\Gamma$ for $\bigcup_{\gamma: \Gamma(\gamma) \neq 0} \gamma \subset \mathscr{H}$. Let us remark that

$\Gamma$ is admissible if and only if $\Gamma(\gamma)=1$ or 0 and the connected components of $\Gamma$ are exactly the $\gamma$ with $\Gamma(\gamma) \neq 0$. Let $A$ and $B$ be two sets (of $\mathscr{H}$ ). We write $A i B$ if for every connected component of $B$ there is a connected component of $A$ such that their union is connected. Caution: $A i B \nRightarrow B i A$. In particular if $B=\gamma$ a contour and $A=\Gamma$ a family of contours, then $\Gamma i \gamma$ means that there is a $\gamma_{1}, \Gamma\left(\gamma_{1}\right) \neq 0$ such that $\gamma_{1} \cup \gamma$ is connected. On the other hand $\gamma i \Gamma$ means that the union of $\gamma$ and each connected component of $\Gamma$ is connected. We also use the notation $\Gamma \subset A_{L, M}$ if $\gamma \subset A_{L, M}$ for every $\gamma$ with $\Gamma(\gamma) \neq 0$.

Let $z=e^{-2 \beta J}$. We define two functions on $\mathscr{L}$ :

$$
\varphi(\Gamma)=\left\{\begin{array}{lc}
z^{|\Gamma|} & \Gamma \text { admissible } \\
0 & \text { otherwise }
\end{array}\right.
$$

and

$$
\varphi^{T}(\Gamma)=\sum_{n=1}^{\infty} \frac{(-1)^{n+1}}{n} \sum^{\prime} \prod_{i=1}^{n} \varphi\left(\Gamma_{i}\right)
$$

where the sum $\sum^{\prime}$ is over all $\Gamma_{1}, \ldots, \Gamma_{n}$ such that $\Gamma_{i} \neq 0$ and $\sum \Gamma_{i}=\Gamma$. We make the convention that an empty sum is zero. Therefore we have $\varphi^{T}(0)=0 . \varphi^{T}(\Gamma)$ is a polynomial in $z$ since $\Gamma_{i} \neq 0$ in (3.1). Furthermore $\varphi(\Gamma)$ and $\varphi^{T}(\Gamma)$ are translation invariant in an obvious manner.

\section{Theorem 2}

(a) $\varphi^{T}(\Gamma) \neq 0 \Rightarrow \Gamma$ is connected;

(b) For any $R>0$ there exists an $r>0$ such that

$$
\sum_{\substack{\Gamma \in \mathscr{L} \\ 0 \in \operatorname{In} T}} R^{|\Gamma|}\left|\varphi^{T}(\Gamma)\right| \leqq \frac{|z|}{1-|z|}, \quad|z|<r
$$

where Int $\Gamma=\bigcup_{\gamma: \Gamma(\gamma) \neq 0} \operatorname{Int} \gamma$.

Remarks. Theorem 2 is standard although the formulation of (b) may not be. 
For a proof see [App. 1 in 5] and [7]. It is easy to see that (b) implies

$$
\sum_{\substack{\Gamma \in \mathscr{L} \\ \Gamma i f}} R^{|\Gamma|}\left|\varphi^{T}(\Gamma)\right| \leqq \frac{|z|}{1-|z|}, \quad|z|<r
$$

where $f$ is some face of $\mathscr{H}$. Using (b) one obtains also that

$$
\sum_{\Gamma \subset A} \varphi^{T}(\Gamma)
$$

is analytic in $z,|z|<r$ and a justification, for $|z|<r$, of the formula

$$
\sum_{\Gamma \subset A} \varphi(\Gamma)=\exp \left(\sum_{\Gamma \subset A} \varphi^{T}(\Gamma)\right)
$$

Here $\Lambda$ is any finite subset of $\mathbb{Z}^{3}$.

Theorem 3. Let $\Lambda$ be a subset of $\mathbb{Z}^{3}$. For any $R>0$ there exists $r>0$ such that for any finite $B \subset \Lambda$ and any $\Gamma$ there exists a function $f_{B}(\Gamma)$ which is analytic in $z,|z|<r$ and such that:

(a) $\left\langle\sigma^{B}\right\rangle_{A}^{+}=\sum_{\Gamma \in A} f_{B}(\Gamma)$

(b) $f_{B}(\Gamma) \neq 0$ implies that

$$
\Gamma=\sum_{i=1}^{n} \Gamma_{i}
$$

for some $\Gamma_{i}, i=1, \ldots, n$, with the properties: $\Gamma_{i}$ is a connected set, $i=1, \ldots, n$; Int $\Gamma_{i} \cap B \neq \varnothing, i=1, \ldots, n$.

(c) $\sum_{\Gamma \in \mathscr{L}}\left|f_{B}(\Gamma)\right| R^{|\Gamma|} \leqq \exp \left(|B| \frac{|z|}{1-|z|}\right)$.

The proof of this theorem is in Appendix 1.

\section{The Interface}

Given a configuration in $A_{L, M}$ with \pm b.c. we consider the set of all faces of $\mathscr{H}$ associated to $\langle i, j\rangle$ with $\sigma_{i} \neq \sigma_{j}$. We decompose this set into maximally connected components. There is exactly one component which is infinite when we extend the configuration to all $\mathbb{Z}^{3}$ using the \pm b.c. We call this component the interface $\Delta$. All other components are contours. For every interface $\Delta$ of a configuration in $A_{L, M}$ there exists exactly one configuration $\sigma_{\Delta}$ which contains $\Delta$ and no contour. As for the contours we use the notation $\Delta \subset A_{L, M}$. A contour $\gamma \subset A_{L, M}$ and $\Delta \subset A_{L, M}$ are compatible if there exists a configuration in $A_{L, M}$ with \pm b.c. containing $\Delta$ as interface and only the contour $\gamma$. This is the case if and only if $\gamma i \Delta(\gamma i \Delta$ is the negation of $\gamma i \Delta)$. Let

$$
X_{\Delta}(\gamma)=\left\{\begin{array}{lll}
1 & \text { if } & \gamma i \Delta \\
0 & \text { if } & \gamma i \Delta
\end{array} .\right.
$$


Let also

$$
X_{\Delta}(\Gamma)=\prod_{\gamma} X_{\Delta}(\gamma)^{\Gamma(\gamma)}
$$

Then we have

$$
Z_{L, M}^{ \pm}=\sum_{\Delta \subset A_{L}, M} z^{|\Delta|} \sum_{\Gamma \subset A_{L}, M} \varphi(\Gamma) X_{\Delta}(\Gamma) .
$$

Since $X_{\Delta}(\Gamma)$ is multiplicative, $X_{\Delta}\left(\Gamma_{1}+\Gamma_{2}\right)=X_{\Delta}\left(\Gamma_{1}\right) X_{\Delta}\left(\Gamma_{2}\right)$,

$$
\sum_{\Gamma \subset A_{L}, M} \varphi(\Gamma) X_{\Delta}(\Gamma)=\exp \left(\sum_{\Gamma \subset A_{L}, M} \varphi^{T}(\Gamma) X_{\Delta}(\Gamma)\right) .
$$

We are going to consider the system in an infinite cylinder of base $L \times L$, $A_{L}=\left\{i \in \mathbb{Z}^{3}:-L \leqq i_{2}, i_{3}<L\right\}$, by taking the limit $M \rightarrow \infty$. This is necessary in order to analyze the interface. Let us prove first the existence of the limit for the ratio of the partition functions

$$
\begin{aligned}
& Z_{L, M}=\frac{Z_{L, M}^{ \pm}}{Z_{L, M}^{+}} \\
& =\frac{\sum_{\Delta \subset A_{L}, M} z^{|\Delta|} \sum_{\Gamma \subset A_{L}, M} \varphi(\Gamma) X_{\Delta}(\Gamma)}{\sum_{\Gamma \subset A_{L}, M} \varphi(\Gamma)} \\
& =\sum_{\Delta \subset A_{L}, M} z^{|\Delta|} \exp \left(\sum_{\Gamma \subset A_{L, M}} \varphi(\Gamma)\left(X_{\Delta}(\Gamma)-1\right)\right) \\
& =\sum_{\Delta \subset A_{L, M}} z^{|\Delta|} \exp \left(-\sum_{\substack{\Gamma \subset A_{L}, M \\
\Gamma i \Delta}} \varphi^{T}(\Gamma)\right) .
\end{aligned}
$$

Using Theorem 2 we have

$$
\sum_{\Gamma \subset A_{L}, M}\left|\varphi^{T}(\Gamma)\right| \leqq|\Delta| \frac{|z|}{1-|z|}, \quad|z|<r
$$

and moreover

$$
\lim _{\substack{M \rightarrow \infty \\ \Gamma \subset A_{L}, M}} \sum_{\substack{\Gamma i \Delta \\ A_{L}}} \varphi^{T}(\Gamma)=\sum_{\substack{\Gamma \subset A_{L} \\ \Gamma i \Delta}} \varphi^{T}(\Gamma), \quad|z|<r .
$$

This shows that

$$
\sum_{\substack{\Gamma \subset A_{L} \\ \Gamma i \Delta}} \varphi^{T}(\Gamma)
$$

is analytic in $z,|z|<r$.

We now use the fact that the number of possible $\Delta$ with $|\Delta|=k$ is smaller than $c_{1}^{k}$ in order to obtain the bound

$$
Z_{L, M} \leqq \sum_{k \geqq 0} c^{k}|z|^{k}<\infty
$$


where

$$
c=c_{1} \exp \left(\frac{|z|}{1-|z|}\right) \text {. }
$$

This shows that $\lim Z_{L, M}=Z_{L}$ exists and that

$$
M \rightarrow \infty
$$

$$
Z_{L}=\sum_{\Delta \subset A_{L}} z^{|\Delta|} \exp \left(-\sum_{\substack{\Gamma \subset A_{L} \\ \Gamma i \Delta}} \varphi^{T}(\Gamma)\right) .
$$

Hence the probability distribution of $\Delta$ in $A_{L, M}$ given by

$$
P_{L, M}(\Delta)=z^{|\Delta|} \exp \left(-\sum_{\substack{\Gamma \subset A_{L, M} \\ \Gamma i \Delta}} \varphi^{T}(\Gamma)\right) \cdot Z_{L, M}^{-1}
$$

has the limit

$$
\lim _{M \rightarrow \infty} P_{L, M}(\Delta)=P_{L}(\Delta)=z^{|\Delta|} \exp \left(-\sum_{\substack{\Gamma \subset A_{L} \\ \Gamma i \Delta}} \varphi^{T}(\Gamma)\right) Z_{L}^{-1} .
$$

We recall now the basic geometrical properties of the interface in $A_{L}$. We refer to Dobrushin [1] for details. Let $\pi=\left\{x \in \mathbb{R}^{3}: x_{1}=-1 / 2\right\}$ be the regular plane and $p(\cdot)$ be the orthogonal projection on $\pi$. Let $\mathscr{P}$ be the set of all projections of the elements of $\mathscr{H}$ on $\pi$. An element of $\mathscr{P}$ is either a face or an edge. Let $\mathscr{P}_{0}=\mathscr{P} \cap \mathscr{H}$ be the set of faces in $\mathscr{P}$. Two elements of $\mathscr{P}$ are adjacent if their union is a connected subset of $\pi$. There are two types of faces in $\Delta:$ The $c$-faces which are the horizontal faces $f$ such that there is no other face $g$ in $\Delta$ with $p(f)=p(g)$. The $w$-faces are all other faces of $\Delta$. The interface $\Delta$ is decomposed into walls which are the connected components of the set of all $w$-faces and into ceilings. (The ceilings are connected subsets but not maximally connected components of the set of all $c$-faces, see [1].)

Let $W_{1}, \ldots, W_{n}$ be the walls of $\Delta$ and $C_{1}, \ldots, C_{m}$ the ceilings of $\Delta$. We project $\Delta$ on $\pi$. Then if $W_{1}$ and $W_{2}$ are different walls of $\Delta$, i.e., they are disconnected, then $p\left(W_{1}\right)$ and $p\left(W_{2}\right)$ are disconnected. Moreover all faces of $\mathscr{P} \backslash p\left(W_{i}\right)$ which are adjacent to $p\left(W_{i}\right)$ are projections of $c$-faces. Let $W$ be a wall. We decompose $\mathscr{P}_{0} \backslash p(W)$ into connected components. The interior of $p(W)$, Int $W$, is the union of the finite components of $\mathscr{P}_{0} \backslash p(W)$ and the exterior Ext $W$ is the infinite component of $\mathscr{P}_{0} \backslash p(W)$. To every component of $\mathscr{P}_{0} \backslash p(W)$ there corresponds one ceiling adjacent to $W$ which projects into this component. The base of $W$ is the ceiling adjacent to $W$ which projects into Ext $W$. We call a standard wall a wall $W$ such that there is an interface $\Delta(W)$ containing only this wall. The base of a standard wall lies in the regular plane. To any wall $W$ of an interface we can associate a standard wall which is just the translate of $W$ in such a way that its base lies in $\pi$. Therefore we can associate to every interface $\Delta$ a family of standard walls such that their projections in $\mathscr{P}$ are disconnected. The converse is also true: for any family $\theta$ of standard walls with disconnected projections we can reconstruct in a 
unique way an interface $\Delta(\theta)$. We say that such a family of standard walls is admissible. The set of all standard walls is denoted by $\mathscr{H}$ and we write $W \subset A_{L}$ if $\Delta(W) \subset A_{L}$. We identify $\theta$ with the function on $\mathscr{H}$ such that $\theta(W)=1$ if $W$ belongs to the family and $\theta(W)=0$ otherwise. As before $N(\theta)=\sum_{W} \theta(W)$. We write also $\theta \subset A_{L}$ if $W \subset A_{L}$ for all $W$ with $\theta(W) \neq 0$. The function identically zero is denoted by 0 . $\theta_{1} \leqq \theta_{2}$ if and only if $\theta_{1}(W) \leqq \theta_{2}(W)$. In this case $\theta_{2}-\theta_{1}$ is well defined. The projection of $\theta$ is $p(\theta)=\bigcup_{W: \theta(W) \neq 0} p(W)$. In the following we omit the word standard when no confusion arises. A wall $W$ of a family $\theta$ is called minimal if there is no other wall $W^{\prime}$ of $\theta$ such that $p\left(W^{\prime}\right) \subset$ Int $W$. Each family $\theta$ has at least one minimal wall. We call external a wall $W$ of $\theta$ if $p\left(W^{\prime}\right) \subset \operatorname{Int} W$ for every $W^{\prime}$ of $\theta$. We define for each $W$ the "excess" number of faces in $W$,

$$
\Pi(W)=|W|-|p(W) \cap \mathscr{H}| .
$$

We notice the inequality

$$
|p(W)| \leqq \Pi(W)
$$

(In Dobrushin [1] $|p(W)|$ denotes $|p(W) \cap \mathscr{H}|$.) It is easy to verify that the total excess area of an interface $\Delta(\theta)$ is $\Pi(\theta)$, i.e., $|\Delta(\theta)|=(2 L)^{2}+\Pi(\theta)$ where $\Pi(\theta)=$ $\sum_{W} \theta(W) \Pi(W)$. We introduce now the function

$$
\varphi_{L}(\theta)=z^{\Pi(\theta)} \exp \left[-\sum_{\substack{\Gamma \subset A_{L} \\ \Gamma i \Delta(\theta)}} \varphi^{T}(\Gamma)+\sum_{\substack{\Gamma \subset A_{L} \\ \Gamma i \mathscr{P}_{0}}} \varphi^{T}(\Gamma)\right]
$$

if $\theta \subset A_{L}$ is admissible and $\varphi_{L}(\theta)=0$ otherwise.

Then we can write

$$
P_{L}(\Delta(\theta))=\frac{\varphi_{L}(\theta)}{\sum_{\theta \subset A_{L}} \varphi_{L}(\theta)} .
$$

The quantity

$$
U_{L}(\theta)=\sum_{\substack{\Gamma \subset A_{L} \\ \Gamma i \Delta(\theta)}} \varphi^{T}(\Gamma)-\sum_{\substack{\Gamma \subset A_{L} \\ \Gamma i \mathscr{P}_{0}}} \varphi^{T}(\Gamma)
$$

is called the effective energy of the family of walls $\theta$. The normalization of $U_{L}(\theta)$ is chosen so that $U_{L}(0)=0$.

We introduce also nonadmissible $\theta$ so that we can use the algebraic formalism.

Let $\mathscr{T}$ be the set of functions $\theta$ on $\mathscr{H}$ with values in $\mathrm{N}$ and such that $N(\theta)=\sum_{W} \theta(W)<\infty$. We extend $\varphi_{L}(\theta)$ on $\mathscr{T}$ by putting $\varphi_{L}(\theta)=0$ if $\theta$ is nonadmissible. We introduce also the function

$$
\varphi_{L}^{T}(\theta)=\sum_{n \geqq 1} \frac{(-1)^{n+1}}{n} \sum_{\theta_{1}, \ldots, \theta_{n}}^{\prime} \prod_{i=1}^{n} \varphi_{L}\left(\theta_{i}\right)
$$

where the prime indicates the restriction in the sum to $\theta_{i} \neq 0, i=1, \ldots, n$ and $\sum \theta_{i}=\theta$. 


\section{Remarks}

(1) Using properties of the $\varphi^{T}(\Gamma)$ one shows that $\varphi_{L}(\theta)$ is analytic in $z$ for $|z|$ small. Since the sum defining $\varphi_{L}^{T}(\theta)$ is in fact finite $\varphi_{L}^{T}(\theta)$ is also analytic in $z$ for $|z|$ small.

(2) The formal expression

$$
\sum_{\theta \subset A_{L}} \varphi_{L}(\theta)=\exp \left(\sum_{\theta \subset A_{L}} \varphi_{L}^{T}(\theta)\right)
$$

is valid for $|z|$ small using the estimations of Theorem 4.

(3) The surface tension in $A_{L}$ is given by

$$
\begin{gathered}
\tau_{L}=-\frac{1}{(2 L)^{2}} \log \left\{\lim _{M \rightarrow \infty} Z_{L, M}\right\} \\
=2 \beta J+\frac{1}{(2 L)^{2}} \sum_{\substack{\Gamma \subset A_{L} \\
\Gamma i \mathscr{P}_{0}}} \varphi^{T}(\Gamma)-\frac{1}{(2 L)^{2}} \sum_{\theta \in A_{L}} \varphi_{L}^{T}(\theta) .
\end{gathered}
$$

We derive now convenient expressions for the correlation functions $\left\langle\sigma^{B}\right\rangle_{L}^{ \pm}$. Let $\Delta=\Delta(\theta)$ be an interface in $A_{L}$. We define $\overline{S(\theta)}$ as the set of sites $i \in \mathbb{Z}^{3}$ such that there exists a face, associated to some $\langle i, j\rangle$, which has a non-empty intersection with $\Delta(\theta)$. If we fix $\Delta(\theta)$ and look at all possible spin configurations in $A_{L}$ with this interface, then $\overline{S(\theta)}$ is exactly the set of sites $i$ where $\sigma_{i}$ has a well-defined value determined by $\Delta(\theta)$. We put $S(\theta)=A_{L} \backslash \overline{S(\theta)}$ and we observe that $\overline{S(\theta)}$ induces for each connected components of $S(\theta)$ a pure boundary condition $\alpha=+$ or $\alpha=-$. We can always take this to be a + b.c. by reversing the spins in some of the connected components of $S(\theta)$. Let now $B$ be a finite subset of $A_{L, M}$ we then have

$$
\begin{aligned}
\left\langle\sigma^{B}\right\rangle_{L}^{ \pm} \equiv \lim _{M \rightarrow \infty}\left\langle\sigma^{B}\right\rangle_{L, M}^{ \pm} & =\sum_{\theta \subset A_{L}} P_{L}(\theta)\left\langle\sigma^{B} \mid \theta\right\rangle_{L} \\
& =\sum_{\theta \subset A_{L}} P_{L}(\theta) \chi_{B}(\theta)\left\langle\sigma^{\bar{B}}\right\rangle_{\theta}
\end{aligned}
$$

where $\left\langle\sigma^{B} \mid \theta\right\rangle_{L}$ is the conditional expectation value of $\sigma^{\bar{B}}$ in $A_{L}$ given $\Delta(\theta)$; $P_{L}(\theta)=P_{L}(\Delta(\theta))$ and $\chi_{B}(\theta)$ is chosen to be +1 or -1 so that

$$
\left\langle\sigma^{B} \mid \theta\right\rangle_{L}=\chi_{B}(\theta)\left\langle\sigma^{\bar{B}}\right\rangle_{\theta}
$$

with $\bar{B}=B \cap S(\theta) .\left\langle\sigma^{\bar{B}}\right\rangle_{\theta}$ is the expectation value of $\sigma^{\bar{B}}$ in $S(\theta)$ with + b.c. Notice that the sum is in fact only on admissible $\theta$ since otherwise $P_{L}(\theta)=0$. Given a family of walls $\theta_{1}$ in $A_{L}$ we define their correlation function as

$$
\rho_{L}\left(\theta_{1}\right)=\frac{\sum_{\theta_{2} \subset A_{L}} \varphi_{L}\left(\theta_{1}+\theta_{2}\right)}{\sum_{\theta_{2} \subset A_{L}} \varphi_{L}\left(\theta_{2}\right)} .
$$

Using (4.1) we have

$$
\rho_{L}\left(\theta_{1}\right)=\sum_{\substack{\theta_{2} \subset A_{L} \\ \theta_{1} \leqq \theta_{2}}} P_{L}\left(\theta_{2}\right) .
$$


since $P_{L}(\theta)=\rho_{L}(\theta)=0$ if $\theta$ is non-admissible we have the formula

$$
P_{L}\left(\theta_{1}\right)=\sum_{\substack{\theta_{2} \subset A_{L} \\ \theta_{1} \leqq \theta_{2}}} \rho_{L}\left(\theta_{2}\right)(-1)^{N\left(\theta_{2}-\theta_{1}\right)} .
$$

Putting $\left\langle\sigma^{B} \mid \theta\right\rangle_{L}=0$ if $\theta$ is non-admissible we have

$$
\begin{aligned}
\left\langle\sigma^{B}\right\rangle_{L}^{ \pm} & =\sum_{\theta \subset A_{L}} P_{L}(\theta)\left\langle\sigma^{B} \mid \theta\right\rangle_{L} \\
& =\sum_{\theta \subset A_{L}} \sum_{\theta^{\prime} \subset A_{L}} \rho_{L}\left(\theta^{\prime}\right)(-1)^{N\left(\theta^{\prime}-\theta\right)}\left\langle\sigma^{B} \mid \theta\right\rangle_{L} \\
& =\sum_{\theta^{\prime} \subset A_{L}} \rho_{L}\left(\theta^{\prime}\right)\left(\sum_{\substack{\theta \subset A_{L} \\
\theta \leqq \theta^{\prime}}}(-1)^{N\left(\theta^{\prime}-\theta\right)}\left\langle\sigma^{B} \mid \theta\right\rangle_{L}\right) .
\end{aligned}
$$

Theorem 4. For any $R>0$ there exists $r>0$ such that if $|z|<r$

(a) $\left|\rho_{L}(\theta)\right| \leqq R^{-\Pi(\theta)} \frac{|z|^{N(\theta)}}{1-|z|}$

and $\rho_{L}(\theta)$ is analytic in $z$.

(b) $\sum_{\text {Int } \theta \ni X_{0}}\left|\varphi_{L}^{T}(\theta)\right| \leqq \frac{|z|}{1-|z|}, \quad|z|<r$

where Int $\theta=\bigcup_{\theta(W) \neq 0}$ Int $W$ and $x_{0} \in \pi$.

Remark. It is in fact possible to show also

$$
\sum_{\substack{\theta \\ \text { Int } \theta \ni x_{0}}} R^{\Pi(\theta)}\left|\varphi_{L}^{T}(\theta)\right| \leqq \frac{|z|}{1-|z|} .
$$

However we do not use such a bound.

\section{The Energy of the Walls}

In this section $A_{L}$ is fixed and we do not write always $\Gamma \subset A_{L}$ or $\theta \subset A_{L}$. The effective energy of an admissible family of walls is defined by (4.2). We rewrite $U_{L}(\theta)$ as a sum of potentials $\Phi_{L}(\theta)$

$$
U_{L}(\theta)=\sum_{\theta^{\prime} \leqq \theta} \Phi_{L}\left(\theta^{\prime}\right)
$$

Notice that since $\theta$ is admissible $\theta^{\prime}$ is also admissible. The Möbius inversion formula yields

$$
\Phi_{L}(\theta)=\sum_{\theta^{\prime} \leqq \theta}(-1)^{N\left(\theta-\theta^{\prime}\right)} U_{L}\left(\theta^{\prime}\right)
$$

Theorem 5 below gives the properties of these potentials which allow us to analyze the distribution $P_{I}(\theta)$. 
is analytic in $z$ for $|z|<r$, and satisfies

$$
\sum_{W \leqq \theta^{\prime} \leqq \theta}\left|\Phi_{L}\left(\theta^{\prime}\right)\right| R^{\delta\left(\theta^{\prime}\right)} \leqq 3 \Pi(W) \frac{|z|}{1-|z|}
$$

where $W$ is any minimal wall of $\theta$ and $\delta(\theta)=\min \{|E|: E$ connected $\subset \mathscr{P}, E \cup p(\theta)$ connected\}.

Proof. By definition

$$
U_{L}(\theta)=\sum_{\Gamma i \Delta(\theta)} \varphi^{T}(\Gamma)-\sum_{\Gamma i \mathscr{P}_{0}} \varphi^{T}(\Gamma)
$$

Suppose that $\Gamma$ is connected (otherwise $\varphi^{T}(\Gamma)=0$ ) and that $\Gamma i \Delta(\theta)$ but $\bar{\theta} i \bar{\Gamma}$. To simplify the notation we have put $\bar{\Gamma}=p(\Gamma)$ and $\bar{\theta}=p(\theta)$. Then from the definition $\Gamma i C$ exactly for one ceiling $C$ of $\Delta(\theta)$. Using the invariance under translation in the $i_{1}$-direction of $\varphi^{T}$ we get

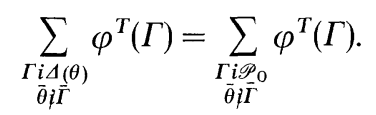

Therefore

$$
U_{L}(\theta)=\sum_{\Gamma i \Delta(\theta)} \varphi^{T}(\Gamma)-\sum_{\substack{\Gamma i \mathscr{P}_{0} \\ \bar{\theta} i \bar{\Gamma}}} \varphi^{T}(\Gamma) .
$$

We treat each sum separately. We define $\Phi_{1, L}(\theta)$ by

$$
\sum_{\theta^{\prime} \leqq \theta} \Phi_{1, L}\left(\theta^{\prime}\right) \equiv U_{1, L}(\theta)=\sum_{\substack{\Gamma i \Delta(\theta) \\ \bar{\theta} i \bar{\Gamma}}} \varphi^{T}(\Gamma) .
$$

By the inversion formula

$$
\begin{aligned}
\Phi_{1, L}(\theta) & =\sum_{0 \neq \theta^{\prime} \leqq \theta}(-1)^{N\left(\theta-\theta^{\prime}\right)} \sum_{\Gamma i \Delta\left(\theta^{\prime}\right)} \varphi^{T}(\Gamma) \\
& =\sum_{\Gamma} \varphi^{T}(\Gamma) \sum_{\theta^{\prime}}^{\prime}(-1)^{N\left(\theta-\theta^{\prime}\right)}
\end{aligned}
$$

where the final sum $\sum^{\prime}$ is over $\theta^{\prime}$ such that $0 \neq \theta^{\prime} \leqq \theta, \Gamma i \Delta\left(\theta^{\prime}\right)$ and $\bar{\theta}^{\prime} i \bar{\Gamma}$ for fixed $\Gamma$.

Let $W$ be a minimal wall of $\theta$. For any $\theta^{\prime}, 0 \neq \theta^{\prime} \leqq \theta$ we consider the connected component of $\mathscr{P}_{0} \backslash\left(\bar{\theta}^{\prime} \backslash \bar{W}\right)$ which contains $\bar{W}$. We denote by $\widetilde{W}\left(\theta^{\prime}\right)$ the part of $\Delta\left(\theta^{\prime}\right)$ which projects on this connected component. For example if $W \notin \theta^{\prime}$, then $\widetilde{W}\left(\theta^{\prime}\right)$ is just a ceiling in $\Delta\left(\theta^{\prime}\right)$ and if $\theta^{\prime}=W, \widetilde{W}\left(\theta^{\prime}\right)=\Delta(W)$. Let us now suppose that we have a $\Gamma$ satisfying the following condition

either $\bar{\Gamma} \downarrow \bar{W}$

or $\bar{\Gamma} i \bar{W}$ and $\Gamma i \widetilde{W}\left(\theta^{\prime}\right)$ for all $\theta^{\prime} \leqq \theta$

For a such a $\Gamma$ we have

$$
\sum_{\theta^{\prime}}^{\prime}(-1)^{N\left(\theta-\theta^{\prime}\right)}=\sum_{0 \neq \theta^{\prime} \leqq \theta-W}^{\prime}(-1)^{N\left(\theta-\theta^{\prime}\right)}+\sum_{0 \neq \theta^{\prime \prime} \leqq \theta-W}^{\prime}(-1)^{N\left(\theta-\theta^{\prime \prime}-W\right)}=0
$$

We have another cancellation when there exists an external wall $W$ in $\theta$. Since $W$ is external it is also a standard wall in $\Delta(\theta)$. If there exists a $\Gamma$ such that $\Gamma i \Delta(\theta)$ 
with $\overline{\theta-W} i \bar{\Gamma}$ and $\Gamma j W$, then there exists a translate $\Gamma^{\prime}$ of $\Gamma$ with $\Gamma^{\prime} i \Delta(\theta-W)$. Therefore we obtain a cancellation. Using these two results we get

$$
\begin{aligned}
\Phi_{1, L}(\theta) & =\sum_{\Gamma} \varphi^{T}(\Gamma) \sum_{\theta^{\prime}}(-1)^{N\left(\theta-\theta^{\prime}\right)} \\
& =\sum_{\bar{I} i \bar{\theta}}^{*} \varphi^{T}(\Gamma) \sum_{\theta^{\prime}}^{\prime}(-1)^{N\left(\theta-\theta^{\prime}\right)}
\end{aligned}
$$

where the sum $\sum^{\prime}$ is as above and the sum $\sum^{*}$ is restricted to $\Gamma$ such that $\Gamma$ intersects the translate $\widetilde{W}\left(\theta^{\prime}\right)$ for some $\theta^{\prime} \leqq \theta$ whenever $W$ is minimal in $\theta$. Since $\bar{W} i \bar{\Gamma}$ for all minimal or external walls $W$ in $\theta$ and since $\Gamma$ is connected we have $\bar{W} i \bar{\Gamma}$ for all $W$ in $\theta$ and therefore $\bar{\Gamma} i \bar{\theta}$. We choose now some minimal wall $W_{0}$ of $\theta$.

$$
\begin{aligned}
\left|\Phi_{1, L}(\theta)\right| & \leqq \sum_{\overline{I i \bar{\theta}}}^{0}\left|\varphi^{T}(\Gamma)\right| 2^{N(\theta)} \\
& \leqq 4^{N(\theta)} \sum_{\substack{\bar{T} i \bar{\theta} \\
\Gamma i \Delta\left(W_{0}\right)}}\left|\varphi^{T}(\Gamma)\right|
\end{aligned}
$$

where $\sum^{0}$ is restricted to $\Gamma$ such that $\Gamma$ intersects $\widetilde{W}_{0}\left(\theta^{\prime}\right)$ for some $\Delta\left(\theta^{\prime}\right), \theta^{\prime} \leqq \theta$. The first inequality comes from counting the number of $\theta^{\prime} \leqq \theta$ and the second inequality comes from the fact that there are at most $2^{N(\theta)}$ different $\widetilde{W}_{0}\left(\theta^{\prime}\right)$ and from the translation invariance of $\varphi^{T}(\Gamma)$.

Using the definition of $\bar{\Gamma} i \bar{\theta}$ and the fact that $\Gamma$ is a contour, i.e., the union of the faces of $\Gamma$ is a closed connected polyhedron, we have the inequality

$$
N(\theta) \leqq|\Gamma| .
$$

Therefore

$$
\left|\Phi_{1, L}(\theta)\right| \leqq \sum_{\substack{\Gamma i \bar{\theta} \\ \Gamma i \Delta\left(W_{0)}\right.}} 4^{|\Gamma|}\left|\varphi^{T}(\Gamma)\right| .
$$

We now observe that for $R>1$

$$
\sum_{W_{0} \leqq \theta^{\prime} \leqq \theta} R^{\delta\left(\theta^{\prime}\right)} \sum_{\substack{\Gamma i \bar{\theta}^{\prime} \\ \Gamma i \Delta\left(W_{0}\right)}}\left|\varphi^{T}(\Gamma)\right| 4^{|\Gamma|} \leqq \sum_{W_{0} \leqq \theta^{\prime} \leqq \theta} \sum_{\substack{\Gamma i \bar{\theta}^{\prime} \\ \Gamma i \Delta\left(W_{0}\right)}}\left|\varphi^{T}(\Gamma)\right|(4 R)^{|\Gamma|} .
$$

Since $\bar{\Gamma} i \bar{\theta}^{\prime}$ and $\bar{\Gamma}$ is connected we have $\delta\left(\theta^{\prime}\right) \leqq|\bar{\Gamma}| \leqq|\Gamma|$. The number of $\theta^{\prime}$ such that $W_{0} \leqq \theta^{\prime} \leqq \theta$ and $\bar{\Gamma} i \bar{\theta}^{\prime}$ is smaller than $2^{|\bar{\Gamma}|}$ since $\theta$ is admissible. Indeed this implies that there is only one wall in $\theta$ with a given projection and all projections of the walls of $\theta$ are disconnected. Therefore if we sum first over $\theta^{\prime}$ and then over $\Gamma$ in (5.1) we have

$$
\sum_{W_{0} \leqq \theta^{\prime} \leqq \theta} R^{\delta\left(\theta^{\prime}\right)}\left|\Phi_{1, L}\left(\theta^{\prime}\right)\right| \leqq \sum_{f \in \Delta\left(W_{0}\right)} \sum_{\Gamma i f}(8 R)^{|\Gamma|}\left|\varphi^{T}(\Gamma)\right| \leqq 2 \Pi\left(W_{0}\right) \frac{|z|}{1-|z|}
$$

if $|z|$ is small enough. The sum is estimated as in (5.2) below.

We estimate now the second part of $U_{L}(\theta)$ :

$$
\begin{aligned}
& U_{2, L}(\theta)=-\sum_{\substack{\Gamma i \mathscr{P}_{0} \\
\bar{\theta}_{\bar{l}} \bar{\Gamma}}} \varphi^{T}(\Gamma) \equiv \sum_{\theta^{\prime} \leqq \theta} \Phi_{2, L}\left(\theta^{\prime}\right) \\
& =\sum_{\theta^{\prime} \leqq \theta}(-1)^{N\left(\theta^{\prime}\right)} \sum_{\substack{\Gamma i \mathscr{\rho}_{0} \\
\bar{\Gamma}: \bar{Q}^{\prime}}} \varphi^{T}(\Gamma) .
\end{aligned}
$$


By identification we get

$$
\Phi_{2, L}(\theta)=(-1)^{N(\theta)} \sum_{\substack{\Gamma i \mathscr{P}_{0} \\ \bar{\Gamma} i \bar{\theta}}} \varphi^{T}(\Gamma)
$$

Therefore

$$
\sum_{W_{0} \leqq \theta^{\prime} \leqq \theta} R^{\delta\left(\theta^{\prime}\right)}\left|\Phi_{2, L}\left(\theta^{\prime}\right)\right| \leqq \sum_{W_{0} \leqq \theta^{\prime} \leqq \theta} \sum_{\substack{\Gamma i \mathscr{P}_{0} \\ \bar{\Gamma} i \bar{\theta}^{\prime}}}\left|\varphi^{T}(\Gamma)\right| R^{|\Gamma|} .
$$

Using the invariance by translation of $\varphi^{T}(\Gamma)$ we get

$$
\sum_{W_{0} \leqq \theta^{\prime} \leqq \theta} \sum_{\substack{\Gamma i \bar{\theta}^{\prime} \\ \Gamma i \bar{W}_{0}}}\left|\varphi^{T}(\Gamma)\right| R^{|\Gamma|}|\Gamma|
$$

Indeed any $\Gamma$ such that $\Gamma i \mathscr{P}_{0}$ and $\bar{\Gamma} i \bar{W}_{0}$ can be translated so that its translate intersects $\mathscr{P}_{0}$ in $\bar{W}_{0}$. We can obtain at most $|\Gamma|$ times the same contour. Therefore we have the upper bound

$$
\sum_{W_{0} \leqq \theta^{\prime} \leqq \theta} \sum_{\substack{\bar{\Gamma} i \overline{\bar{\theta}}^{\prime} \\ \Gamma i \overline{\bar{W}_{0}}}}\left|\varphi^{T}(\Gamma)\right|(2 R)^{|\Gamma|} \leqq \Pi\left(W_{0}\right) \frac{|z|}{1-|z|}
$$

This finishes the proof.

Remark. Theorem 5 shows also that if $\theta$ is admissible and if $W$ is any wall of $\theta$, then we have

$$
\sum_{\substack{W \leqq \theta^{\prime} \leqq \theta \\ W \text { minimal in } \theta^{\prime}}}\left|\Phi\left(\theta^{\prime}\right)\right| R^{\delta\left(\theta^{\prime}\right)} \leqq 3 \Pi(W) \frac{|z|}{1-|z|} .
$$

\section{Proof of Theorem 1}

(a) We know from (4.3) that

$$
\tau_{L}-2 \beta J=\frac{1}{L^{2}} \sum_{\substack{\Gamma \subset A_{L} \\ \Gamma i \mathscr{P}_{0}}} \varphi^{T}(\Gamma)-\frac{1}{L^{2}} \sum_{\theta \subset A_{L}} \varphi^{T}(\theta) .
$$

By Theorem 2 we have for $|z|<r$

$$
\begin{aligned}
\sum_{\substack{\Gamma \subset A_{L} \\
\Gamma i \mathscr{P}_{0}}}\left|\varphi^{T}(\Gamma)\right| & \leqq L^{2} \sum_{0 \in \operatorname{In} t \Gamma}\left|\varphi^{T}(\Gamma)\right| \\
& \leqq L^{2} \frac{|z|}{1-|z|}
\end{aligned}
$$

and by Theorem 4

$$
\begin{aligned}
\sum_{\theta \subset A_{L}}\left|\varphi_{L}^{T}(\theta)\right| & \leqq L^{2} \sum_{x_{0} \in \operatorname{Int} \theta}\left|\varphi_{L}^{T}(\theta)\right| \\
& \leqq L^{2} \frac{|z|}{1-|z|}
\end{aligned}
$$


From this we conclude that $\tau_{L}-2 \beta J$ is analytic in $z,|z|<r$, since it is equal to a uniformly bounded absolutely convergent series of analytic functions. Moreover the bound is uniform in $L$ and we have convergence for $L \rightarrow \infty$ when $\beta J$ is real [9]. This finishes the proof of (a).

(b) From Sect. 4 (4.4) we have

$$
\left\langle\sigma^{B}\right\rangle_{L}^{ \pm}=\sum_{\theta \subset A_{L}} \sum_{\substack{\theta \\ \theta \\ \theta \leqq A_{L}}} \rho_{L}\left(\theta^{\prime}\right)(-1)^{N\left(\theta^{\prime}-\theta\right)}\left\langle\sigma^{B} \mid \theta\right\rangle_{L} .
$$

The right hand side of this equation is analytic in $z$, for $|z|$ small, because

$$
\left\langle\sigma^{B} \mid \theta\right\rangle_{L}=\chi_{B}(\theta)\left\langle\sigma^{\bar{B}}\right\rangle_{\theta}
$$

is an expectation value in the pure phase times a constant and moreover $\rho_{L}(\theta)$ is analytic in $z$ by Theorem 4 .

We are going to show that this series is actually uniformly bounded in $L$ and then we conclude as in (a). Let $\Xi_{B}$ be the set of $\theta$ such that $p(B) \cap \operatorname{Int} W \neq \varnothing$ for every $W$ of $\theta$ and $\Xi_{B}^{*}$ the set of $\theta$ such that $p(B) \cap \operatorname{Int} W=\varnothing$ for every $W$ of $\theta$. Every $\theta$ can be decomposed into $\theta_{1}+\theta_{2}$ with $\theta_{1} \in \Xi_{B}$ and $\theta_{2} \in \Xi_{B}^{*}$. We notice that $\chi_{B}(\theta)=$ $\chi_{B}\left(\theta_{1}\right)$ since $\theta_{1}$ determines completely the part of $\Delta\left(\theta_{1}+\theta_{2}\right)$ which projects itself on $p(B)$. Hence we may write

$$
\begin{aligned}
\left\langle\sigma^{B}\right\rangle_{L}^{ \pm}= & \sum_{\theta \in \Xi_{B}} \sum_{\theta^{\prime} \in E_{B}^{*}} \rho_{L}\left(\theta+\theta^{\prime}\right) \sum_{\theta_{1} \leqq \theta} \chi_{B}\left(\theta_{1}\right)(-1)^{N\left(\theta-\theta_{1}\right)} . \\
& \cdot \sum_{\theta_{2} \leqq \theta^{\prime}}(-1)^{N\left(\theta^{\prime}-\theta_{2}\right)}\left\langle\sigma^{\bar{B}}\right\rangle_{\theta_{1}+\theta_{2}} .
\end{aligned}
$$

Let $\theta_{1}$ be fixed. We then have, using Theorem 3

$$
\begin{aligned}
& \sum_{\theta_{2} \leqq \theta^{\prime}}(-1)^{N\left(\theta^{\prime}-\theta_{2}\right)}\left\langle\sigma^{\bar{B}}\right\rangle_{\theta_{1}+\theta_{2}}=\sum_{\theta_{2} \leqq \theta^{\prime}}(-1)^{N\left(\theta^{\prime}-\theta_{2}\right)} \sum_{\Gamma \subset S\left(\theta_{1}+\theta_{2}\right)} f_{\bar{B}}(\Gamma) \\
& \quad=\sum_{\Gamma \subset A_{L}} f_{\bar{B}}(\Gamma) \sum_{\substack{\theta_{2} \leqq \theta^{\prime} \\
\Gamma \subset S\left(\theta_{1}+\theta_{2}\right)}}(-1)^{N\left(\theta^{\prime}-\theta_{2}\right)}
\end{aligned}
$$

where an empty sum is equal to zero. Consider now those terms in the above sum for which $f_{\bar{B}}(\Gamma) \neq 0$. We are going to show that

$$
\sum_{\substack{\theta_{2} \leqq \theta^{\prime} \\ \Gamma \subset S\left(\theta_{1}+\theta_{2}\right)}}(-1)^{N\left(\theta^{\prime}-\theta_{2}\right)}
$$

is nonzero only if $\Gamma$ is large, i.e., $p(\Gamma) i p\left(\theta^{\prime}\right)$.

By Theorem 3 we can write

$$
\Gamma=\sum_{i=1}^{n} \Gamma_{i}
$$

with $\Gamma_{i}$ connected and Int $\Gamma_{i} \cap \bar{B} \neq \varnothing$. Let us suppose that there exists a wall $W$ in $\theta^{\prime}$ such that $p(\Gamma) i p(W)$. Since Int $W \cap p(B)=\varnothing$ and Int $\Gamma_{i} \cap \bar{B} \neq \varnothing$ for any $i=1, \ldots, n p(\Gamma) \cap$ Int $W=\varnothing$. This implies that the presence and absence of $W$ in $\theta_{2}$ does not affect the condition $\Gamma \subset S\left(\theta_{1}+\theta_{2}\right)$ and hence that

$$
\sum_{\theta_{2} \leqq \theta^{\prime}}(-1)^{N\left(\theta^{\prime}-\theta_{2}\right)}=0 .
$$


Thus to have a nonzero contribution to the sum (6.1) we must have $p(\Gamma) i p(W) \forall W$ in $\theta^{\prime}$, i.e., $p(\Gamma) i p\left(\theta^{\prime}\right)$. Let us label also by $p(B)$ the set of faces of $\mathscr{P}_{0}$ which contains the projection of $B$ on the regular plane. Observing that each contour $\gamma$ is a closed polyhedron we have in the case $f_{\bar{B}}(\Gamma) \neq 0$ that $p(\bar{B}) \subset p(\Gamma)$ and $p(B) \cap p\left(\Gamma_{i}\right) \neq \varnothing$ for all $i=1, \ldots, n$. Since $p(\Gamma) i p\left(\theta^{\prime}\right)$ we have $\delta\left(p(B) \cup p\left(\theta^{\prime}\right)\right) \leqq|\Gamma|+\delta(p(B))$.

$$
\begin{aligned}
\left|\sum_{\theta_{2} \leqq \theta^{\prime}}(-1)^{N\left(\theta^{\prime}-\theta_{2}\right)}\left\langle\sigma^{\bar{B}}\right\rangle_{\theta_{1}+\theta_{2}}\right| & \leqq \sum_{\Gamma}\left|f_{\bar{B}}(\Gamma)\right| 2^{N\left(\theta^{\prime}\right)} \\
& \leqq 2^{N\left(\theta^{\prime}\right) i p\left(\theta^{\prime}\right)} R^{\delta(p(B))} R^{-\delta\left(p\left(\theta^{\prime}\right) \cup p(B)\right)} \sum_{\Gamma}\left|f_{\bar{B}}(\Gamma)\right| R^{|\Gamma|} \\
& \leqq 2^{N\left(\theta^{\prime}\right)} R^{\delta(p(B))} R^{-\delta\left(p\left(\theta^{\prime}\right) \cup p(B)\right)} \exp \left(|B| \frac{|z|}{1-|z|}\right)
\end{aligned}
$$

for $|z|$ small. Using Theorem 4 and $N(\theta) \leqq \Pi(\theta)$ we get

$$
\begin{aligned}
\left|\left\langle\sigma^{B}\right\rangle_{L}\right| \leqq & \exp \left(|B| \frac{|z|}{1-|z|}\right) R^{\delta(p(B))} . \\
& \cdot \sum_{\theta^{\prime} \in \Xi_{B}^{*}} \sum_{\theta \in \Xi_{B}} 2^{\Pi(\theta)} 2^{\Pi\left(\theta^{\prime}\right)}\left|\rho_{L}\left(\theta+\theta^{\prime}\right)\right| R^{-\delta\left(p\left(\theta^{\prime}\right) \cup p(B)\right)} \\
\leqq & \exp \left(|B| \frac{|z|}{1-|z|}\right) R^{\delta(p(B))} \frac{|z|}{1-|z|} \sum_{\theta \in \Xi_{B}}(R / 2)^{-\Pi(\theta)} \\
& \cdot \sum_{\theta^{\prime} \in \Xi_{B}^{*}}(R / 2)^{-\Pi\left(\theta^{\prime}\right)} R^{-\delta\left(p\left(\theta^{\prime}\right) \cup p(B)\right)}
\end{aligned}
$$

We estimate the last sum by

$$
\begin{aligned}
\sum_{\theta \in \Xi_{B}}(R / 2)^{-\Pi(\theta)} & \leqq \sum_{n \geqq 0} \frac{1}{n !}\left(\sum_{\operatorname{Int} W \cap B \neq \varnothing} R^{-\Pi(W)}\right)^{n} \\
& \leqq \exp \left(|B| C_{R}\right) \\
C_{R} \rightarrow 0 \text { as } R \rightarrow \infty &
\end{aligned}
$$

On the other hand

$$
\sum_{\theta^{\prime} \in \Xi_{B}^{*}}(R / 2)^{-\Pi\left(\theta^{\prime}\right)} R^{-\delta\left(p\left(\theta^{\prime}\right) \cup p(B)\right)}<\infty .
$$

Indeed

$$
\sum_{\substack{\theta^{\prime} \in \Xi_{B}^{*} \\\left(p\left(\theta^{\prime}\right) \cup p(B)\right)=\ell}}(R / 2)^{-\Pi\left(\theta^{\prime}\right)} \leqq \sum_{n \geqq 0} \frac{1}{n !}\left(\sum_{W}^{*}(R / 2)^{-\Pi(W)}\right)^{n}
$$

where $\sum^{*}$ is the sum over all $W$ which are connected to a set with $\ell$ elements. Therefore we get the bound $\exp \left(C_{R} \ell\right)$. Now we sum over all connected sets which connects $p(B)$. But the number of connected sets with $\ell$ elements and connecting $p(B)$ is smaller that $|B| C_{3}^{\ell}$ for some constant. Therefore $\left\langle\sigma^{B}\right\rangle_{L} \leqq K_{B}$ uniformly in $L$. 


\section{Appendix 1}

Proof of Theorem 3. We define a function on $\mathscr{C}$ by

$$
\sigma_{i}(\gamma)=\left\{\begin{array}{l}
-1 \text { if } i \in \operatorname{Int} \gamma \\
+1 \text { if } i \in \operatorname{Ext} \gamma
\end{array} i \in \mathbb{Z}^{3} .\right.
$$

We put

$$
\begin{aligned}
\sigma_{i}(\Gamma) & =\prod_{\gamma \in \Gamma} \sigma_{i}(\gamma)^{\Gamma(\gamma)} \\
\sigma^{B}(\Gamma) & =\prod_{i \in B} \sigma_{i}(\Gamma) .
\end{aligned}
$$

Let $\Lambda \subset \mathbb{Z}^{3}$ be finite, then

$$
\left\langle\sigma^{B}\right\rangle_{\Lambda}^{+}=\frac{\sum_{\Gamma=\Lambda} \varphi(\Gamma) \sigma^{B}(\Gamma)}{\sum_{\Gamma \subset \Lambda} \varphi(\Gamma)} .
$$

Since $\sigma^{B}(\Gamma)$ is multiplicative

$$
\sum_{\Gamma \subset \Lambda} \varphi(\Gamma) \sigma^{B}(\Gamma)=\exp \left[\sum_{\Gamma \subset \Lambda} \varphi^{T}(\Gamma) \sigma^{B}(\Gamma)\right] .
$$

Therefore

$$
\begin{aligned}
\left\langle\sigma^{B}\right\rangle_{A}^{+} & =\exp \left[\sum_{\Gamma \subset A} \varphi^{T}(\Gamma)\left(\sigma^{B}(\Gamma)-1\right)\right] \\
& =\exp -\left(\sum_{\sigma^{B}(\Gamma)=-1} 2 \varphi^{T}(\Gamma)\right)
\end{aligned}
$$

Using the bound

$$
\sum_{\substack{\Gamma \subset A \\ \sigma^{B}(\Gamma)=-1}}\left|\varphi^{T}(\Gamma)\right| \leqq|B| \sum_{0 \in \operatorname{Int} \Gamma}\left|\varphi^{T}(\Gamma)\right| \leqq|B| \frac{|z|}{1-|z|}
$$

and standard arguments we can prove the formula (A-1) for $\Lambda$ infinite. We have also a uniform bound on $\left|\left\langle\sigma^{B}\right\rangle_{A}^{+}\right|$and using Vitali's Theorem we get the analyticity of $\left\langle\sigma^{B}\right\rangle_{A}^{+}$in $z,|z|\left\langle r\right.$. Let us now expand $\left\langle\sigma^{B}\right\rangle_{A}^{+}$.

$$
\begin{aligned}
\left\langle\sigma^{B}\right\rangle_{\Lambda}^{+} & =\exp \left(-2 \sum_{\substack{\Gamma \subset A \\
\sigma^{B}(\Gamma)=-1}} \varphi^{T}(\Gamma)\right) \\
& =\sum_{n \geqq 0} \frac{(-2)^{n}}{n !}\left(\sum_{\substack{\Gamma \subset A \\
\sigma^{B}(\Gamma)=-1}} \varphi^{T}(\Gamma)\right)^{n} \\
& =1+\sum_{n \geqq 1} \frac{(-2)^{n}}{n !} \sum_{\Gamma \subset A} \sum_{\substack{\sum_{\sigma^{B}(\Gamma, Y=-1} \Gamma_{i}=\Gamma \\
i=1}}^{n} \prod^{T}\left(\Gamma_{i}\right)
\end{aligned}
$$


We define

$$
\begin{aligned}
f_{B}(\Gamma) & =\sum_{n \geqq 1} \frac{(-2)^{n}}{n !} \sum_{\substack{n \\
\sum^{B} \Gamma_{i}=\Gamma}} \prod_{i=1}^{n} \varphi^{T}\left(\Gamma_{i}\right), \quad \Gamma \neq 0 \\
f_{B}(0) & =1 .
\end{aligned}
$$

All rearrangements in the sums are justified using Theorem 2. The sum defining $f_{B}(\Gamma)$ is finite since $\varphi^{T}(0)=0$ and therefore $f_{B}(\Gamma)$ is a polynomial in $z$.

If $f_{B}(\Gamma) \neq 0$ then one of the terms in (A-2) must be nonzero and therefore

$$
\Gamma=\sum_{i=1}^{n} \Gamma_{i}
$$

$\Gamma_{i}$ connected since otherwise $\varphi^{T}\left(\Gamma_{i}\right)=0$ and Int $\Gamma_{i} \cap B \neq \varnothing$ since otherwise $\sigma^{B}\left(\Gamma_{i}\right)=+1$. Finally

$$
\begin{aligned}
\sum_{\Gamma}\left|f_{B}(\Gamma)\right| R^{|\Gamma|} & \leqq 1+\sum_{\Gamma} \sum_{n \geqq 1} \frac{(+2)^{n}}{n !} \sum_{\substack{\sum_{\Gamma_{i}=\Gamma} \\
\sigma^{B}\left(\Gamma_{\imath}\right)=-1}} \prod_{i=1}^{n}\left|\varphi^{T}\left(\Gamma_{i}\right)\right| R^{\left|\Gamma_{i}\right|} \\
& \leqq 1+\sum_{n \geqq 1} \frac{(+2)^{n}}{n !}\left(\sum_{\substack{\Gamma \\
\sigma^{B}(\Gamma)=-1}}\left|\varphi^{T}(\Gamma)\right| R^{|\Gamma|}\right)^{n} \\
& \leqq \exp \left(2|B| \sum_{\substack{\Gamma \\
0 \in \operatorname{Int} T}} R^{|\Gamma|}\left|\varphi^{T}(\Gamma)\right|\right) \\
& \leqq \exp \left(2|B| \frac{|z|}{1-|z|}\right) .
\end{aligned}
$$

Remark. The formula

$$
\left\langle\sigma^{B}\right\rangle_{\Lambda}^{+}=\exp \left[-2 \sum_{\substack{\Gamma \subset A \\ \sigma^{B}(\Gamma)=-1}} \varphi^{T}(\Gamma)\right]
$$

together with Theorem 2 shows that

$$
\frac{\left\langle\sigma^{B} \sigma^{C}\right\rangle_{A}^{+}}{\left\langle\sigma^{B}\right\rangle_{\Lambda}^{+}\left\langle\sigma^{C}\right\rangle_{A}^{+}}-1
$$

is exponentially small with the distance between $B$ and $C$, uniformly in $A$.

It is remarkable that the only ingredients of the proof of Theorem 3 are Theorem 2 and the multiplicative property

$$
\sigma_{i}(\Gamma)=\prod_{\gamma \in \Gamma} \sigma_{i}(\gamma)^{\Gamma(\gamma)}
$$

Now it can be seen from the proof of Theorem 2, see e.g., [7] that the same expansion can be made for the contours of any ferromagnetic spin $1 / 2$ system with finite range interactions provided that this system satisfies the decomposition property (see [11]).

The multiplicative property also holds trivially for these systems. On the other 
hand, it has been shown [11] that spin $1 / 2$ systems on $\mathbb{Z}^{d}$ can be reduced to a system with the decomposition property in the following sense: all correlation functions with + b.c. either vanish or are equal to some correlation functions with + b.c. in the reduced system.

It follows then that all correlation functions of any spin $1 / 2$ ferromagnetic system on $\mathbb{Z}^{d}$ with finite range interactions are analytic in $e^{-2 \beta J(B)}$ for $\beta$ large, where $J(B)$ is the coupling of the bond $B$. Notice that this extends previous results obtained with Asano contractions because these were restricted to the correlation functions generated by the bonds. For higher spin systems or systems on different lattices than $\mathbb{Z}^{d}$, the above results hold for systems with the decomposition property and, with the results of [12], presumably for all systems.

\section{Appendix 2}

Proof of Theorem 4. We refer to [7], Sect. 4 for an exposition of the algebraic formalism. Let $\psi_{1}$ and $\psi_{2}$ be two functions on $\mathscr{T}$. We define a commutative multiplication by

$$
\psi_{1} * \psi_{2}(\theta)=\sum_{\theta_{1} \leqq \theta} \psi_{1}\left(\theta-\theta_{1}\right) \psi_{2}\left(\theta_{1}\right)
$$

Let $\psi$ be such that $\psi(0)=0$. We define an exponential by

$$
\exp \psi=1+\sum_{n \geqq 1} \frac{1}{n !} \psi * \ldots * \psi
$$

where $1(\theta)=0, \theta \neq 0$ and $1(0)=1$. We have

$$
(\exp \psi)(\theta)=1(\theta)+\sum_{n \geqq 1} \frac{1}{n !} \sum_{\sum \theta_{l}=\theta} \psi\left(\theta_{1}\right) \ldots \psi\left(\theta_{n}\right)
$$

$\exp \psi$ is a function on $\mathscr{T}$ such that $\exp \psi(0)=1$. For all such functions we can define the inverse of the exponential, the logarithm, by

$$
\begin{aligned}
\log \psi(\theta) & =\log \left(\mathbb{1}+\psi_{0}\right)(\theta) \\
& =\sum_{n \geqq 1} \frac{(-1)^{n+1}}{n} \sum_{\sum \theta_{l}=\theta} \psi_{0}\left(\theta_{1}\right) \ldots \psi_{0}\left(\theta_{n}\right)
\end{aligned}
$$

with $\psi_{0}(0)=0$. Formally we have $\log \exp \psi=\psi$.

Let

$$
\varphi(\theta)= \begin{cases}z^{I(\theta)} \exp \left(-U_{L}(\theta)\right) & \theta \text { admissible } \\ 0 & \text { otherwise }\end{cases}
$$

with $U_{L}(\theta)$ defined in (4.2). From now on we suppress the index $L$. We see that

$$
\varphi=\exp \varphi^{T}
$$

and that

$$
\varphi^{-1}=\exp \left(-\varphi^{T}\right) .
$$

These expressions are true not only formally but also analytically for $|z|$ small. 
This is a consequence of the bounds we derive in this Appendix.

Let

$$
\Delta_{\theta}\left(\theta^{\prime}\right)=\sum_{\theta_{2} \leqq \theta^{\prime}} \varphi^{-1}\left(\theta^{\prime}-\theta_{2}\right) \varphi\left(\theta+\theta_{2}\right) .
$$

We observe that $\Delta_{\theta}\left(\theta^{\prime}\right)=0$ if $\theta$ is not admissible. The quantity $\Delta_{\theta}\left(\theta^{\prime}\right)$ satisfies a recurrence equation which implies very strong bounds on $\Delta_{\theta}\left(\theta^{\prime}\right)$. To see this we choose one minimal wall $W$ in $\theta$. We write $\theta$ adm. for $\theta$ admissible and rewrite $U\left(\theta+\theta^{\prime}\right)$, for $\theta+\theta^{\prime}$ adm., in the following way

$$
U\left(\theta+\theta^{\prime}\right)=U_{1}(\theta)+U\left(\theta+\theta^{\prime}-W\right)+I\left(\theta, \theta^{\prime}\right)
$$

where

$$
\begin{aligned}
U_{1}(\theta) & =\sum_{W \leqq \theta_{1} \leqq \theta} \Phi\left(\theta_{1}\right) \\
I\left(\theta, \theta^{\prime}\right) & =\sum_{0 \neq \theta_{2} \leqq \theta^{\prime}} V\left(\theta_{2} \theta_{2}\right) \\
V\left(\theta, \theta_{2}\right) & =\sum_{W \leqq \theta_{1} \leqq \theta} \Phi\left(\theta_{1}+\theta_{2}\right) .
\end{aligned}
$$

In what follows we need to expand

$$
\begin{aligned}
\exp \left(-I\left(\theta, \theta^{\prime}\right)\right) & =\prod_{0 \neq \theta_{2} \leqq \theta^{\prime}}\left(\exp \left(-V\left(\theta, \theta_{2}\right)\right)-1+1\right) \\
& =\sum_{n \geqq 0} \frac{1}{n !} \sum_{\substack{0 \neq \theta_{i} \leqq \theta^{\prime} \\
\theta_{i} \neq \theta_{j} \forall i, j}} \prod_{i=1}^{n}\left(e^{-V\left(\theta, \theta_{\imath}\right)}-1\right) \\
& =\sum_{\theta^{\prime \prime} \leqq \theta^{\prime}} \sum_{n \geqq 0} \frac{1}{n !} \sum^{\prime} \prod_{i=1}^{n}\left(e^{-V\left(\theta, \theta_{i}\right)}-1\right)
\end{aligned}
$$

where the sum $\sum^{\prime}$ is over all $\theta_{1}, \ldots, \theta_{n}$ such that

$$
0 \neq \theta_{i} \leqq \theta^{\prime \prime}, \quad \theta_{i} \neq \theta_{j} \forall i, j, \sum_{i=1}^{n} \theta_{i} \geqq \theta^{\prime \prime}
$$

We define $K\left(\theta, \theta^{\prime \prime}\right)$ for $\theta^{\prime \prime} \neq 0$ by

$$
\sum_{n \geqq 1} \frac{1}{n !} \sum^{\prime} \prod_{i=1}^{n}\left(e^{-V\left(\theta, \theta_{i}\right)}-1\right)
$$

where the sum $\sum^{\prime}$ is over all $\theta_{1}, \ldots, \theta_{n}$ which satisfy $(\mathrm{A}-3)$ and we put $K\left(\theta, \theta^{\prime \prime}\right)=1$ if $\theta^{\prime \prime}=0$.

Lemma. We have the equation:

$$
\begin{aligned}
\Delta_{\theta}\left(\theta^{\prime}\right)= & z^{\Pi(W)} e^{-U_{1}(\theta)} \sum_{\substack{\theta^{\prime \prime} \leqq \theta^{\prime} \\
\theta+\theta^{\prime \prime} \text { adm. }}} K\left(\theta, \theta^{\prime \prime}\right) . \\
& \cdot \sum_{\substack{\theta_{3} \leqq \theta^{\prime}-\theta^{\prime \prime} \\
\theta_{3} \text { adm. } \\
\text { Wi } \theta_{3}}}(-1)^{N\left(\theta_{3}\right)} \Delta_{\theta^{\prime \prime}+\theta+\theta_{3}-W}\left(\theta^{\prime}-\left(\theta^{\prime \prime}+\theta_{3}\right)\right) \\
& -
\end{aligned}
$$


where $\theta$ is admissible and $W$ is a minimal wall of $\theta$. We sum over $\theta_{3}=0$.

See Gallavotti [5] Appendix 5 for a proof.

Let

$$
I_{m}^{R}=\sup _{\theta}\left(\sum_{\substack{\theta^{\prime} \\ N\left(\theta+\theta^{\prime}\right)=m}}\left|\Delta_{\theta}\left(\theta^{\prime}\right)\right| R^{\Pi(\theta)}\right)
$$

Since the equation for $\Delta_{\theta}\left(\theta^{\prime}\right)$ relates $\Delta_{\theta}\left(\theta^{\prime}\right)$ with $N\left(\theta+\theta^{\prime \prime}\right)=m+1$ and $\Delta_{\theta_{1}}\left(\theta_{2}\right)$ with $N\left(\theta_{1}+\theta_{2}\right)=m$ it is possible to prove

Lemma. For $R$ large enough there exists an $r_{2}>0$ such that $I_{m+1}^{R} \leqq|z| I_{m}^{R}$ if $|z|<r_{2}$. Proof.

$$
\begin{aligned}
& \sum_{\substack{\theta^{\prime}: \\
N\left(\theta+\theta^{\prime}\right)=m+1}}\left|\Delta_{\theta}\left(\theta^{\prime}\right)\right| R^{\Pi(\theta)} \leqq(|z| R)^{\Pi(W)} e^{\left|U_{1}(\theta)\right|} \\
& \cdot \sum_{\substack{\theta^{\prime}: \\
N\left(\theta+\theta^{\prime}-W\right)=m}} \sum_{\substack{\theta^{\prime \prime} \leq \theta^{\prime} \\
\theta+\theta^{\prime \prime} \text { adm. }}}\left|K\left(\theta, \theta^{\prime \prime}\right)\right| R^{-\Pi\left(\theta^{\prime \prime}\right)} \\
& \cdot \sum_{\substack{\theta_{3} \text { adm. } \\
W i \theta_{3}}} R^{-\Pi\left(\theta_{3}\right)} R^{+\Pi\left(\theta+\theta^{\prime \prime}+\theta_{3}-W\right)}\left|\Delta_{\theta^{\prime \prime}+\theta+\theta_{3}-W}\left(\theta^{\prime}-\left(\theta^{\prime \prime}+\theta_{3}\right)\right)\right| \\
& \leqq I_{m}^{R}\left[(|z| R)^{\Pi(W)} e^{\left|U_{1}(\theta)\right|} \sum_{\substack{\theta^{\prime \prime} \\
\theta+\theta^{\prime \prime} \text { adm. }}}\left|K\left(\theta, \theta^{\prime \prime}\right)\right| R^{-\Pi\left(\theta^{\prime \prime}\right)} \sum_{\substack{\theta_{3} \text { adm. } \\
W i \theta_{3}}} R^{-\Pi\left(\theta_{3}\right)}\right] .
\end{aligned}
$$

We have to take the sup over $\theta$ for these square brackets. Two estimations are easy:

(a) $\left|U_{1}(\theta)\right| \leqq \sum_{W \leqq \theta_{1} \leqq \theta}\left|\Phi\left(\theta_{1}\right)\right| \leqq 3 \Pi(W) \frac{|z|}{1-|z|}$ for $|z|$ small enough by Theorem 5 .

(b) $\begin{aligned} \sum_{\substack{\theta_{3} \mathrm{adm} \\ W i \theta_{3}}} R^{-\Pi\left(\theta_{3}\right)} & \leqq \sum_{n} \frac{1}{n !} \sum_{W i W_{j}, \forall j} \prod_{j} R^{-\Pi\left(W_{j}\right)} \\ & =\sum_{n} \frac{1}{n !}\left(\sum_{W i W^{\prime}} R^{-\Pi\left(W^{\prime}\right)}\right)^{n} \leqq \exp (\Pi(W) C)\end{aligned}$

where $C$ is a constant.

It remains to estimate

$$
\begin{aligned}
& \sum_{\substack{\theta^{\prime \prime}: \\
\theta+\theta^{\prime \prime} \text { adm. }}}\left|K\left(\theta, \theta^{\prime \prime}\right)\right| R^{-\Pi\left(\theta^{\prime \prime}\right)} \\
& \leqq 1+\sum_{\substack{0 \neq \theta^{\prime \prime} \\
\theta+\theta^{\prime \prime} \text { adm. }}} \sum_{n=1}^{\infty} \frac{1}{n !} \sum^{\prime} \prod_{i=1}^{n}\left|e^{-V\left(\theta, \theta_{l}\right)}-1\right| R^{-\Pi\left(\theta^{\prime \prime}\right)}
\end{aligned}
$$

where the sum $\sum^{\prime}$ is over all $\theta_{1}, \ldots, \theta_{n}$ which satisfy (A-3). We use the inequality

$$
\left|e^{-V\left(\theta, \theta_{l}\right)}-1\right| \leqq\left|V\left(\theta, \theta_{i}\right)\right| R^{\delta\left(W+\theta_{\imath}\right)} e^{\left|V\left(\theta, \theta_{l}\right)\right|} R^{-\delta\left(W+\theta_{i}\right)}
$$


and therefore we have to estimate

$$
\sum_{0 \neq \theta_{i} \leqq \theta^{\prime \prime}}\left|V\left(\theta, \theta_{i}\right)\right| R^{\delta\left(W+\theta_{i}\right)} \leqq \sum_{0 \neq \theta_{i} \leqq \theta^{\prime \prime}} \sum_{W \leqq \theta_{3} \leqq \theta}\left|\Phi\left(\theta_{3}+\theta_{i}\right)\right| R^{\delta\left(W+\theta_{i}\right)}
$$

Since $\theta+\theta^{\prime \prime}$ is adm. $\theta_{i}+\theta_{3}$ is adm. Therefore $\delta\left(W+\theta_{i}\right) \leqq \delta\left(\theta_{i}+\theta_{3}\right)$ because $W \leqq \theta_{3}$. Thus we get the upper bound

$$
\sum_{W \leqq \theta_{1} \leqq \theta+\theta^{\prime \prime}}\left|\Phi\left(\theta_{1}\right)\right| R^{\delta\left(\theta_{1}\right)}
$$

However we cannot apply Theorem 5 since $W$ is minimal in $\theta$ but not necessarily in $\theta+\theta^{\prime \prime}$. Let $W_{1}, \ldots, W_{p}$ be the walls in $\theta^{\prime \prime}$ such that $W$ is external for these walls.

$$
\begin{aligned}
\sum_{W \leqq \theta_{1} \leqq \theta+\theta^{\prime \prime}}\left|\Phi\left(\theta_{1}\right)\right| R^{\delta\left(\theta_{1}\right)} \leqq & \sum_{i=1}^{p} \sum_{\substack{W_{i} \leqq \theta_{1} \leqq \theta+\theta^{\prime \prime} \\
W_{i} \text { minimal in } \theta_{1}}}\left|\Phi\left(\theta_{1}\right)\right| R^{\delta\left(\theta_{1}\right)} \\
& +\sum_{\substack{W \leqq \theta_{1} \leqq \theta+\theta^{*} \\
W \text { minımal in } \theta_{1}}}\left|\Phi\left(\theta_{1}\right)\right| R^{\delta\left(\theta_{1}\right)} \\
\leqq & 3 \frac{|z|}{1-|z|}\left(\Pi(W)+\sum_{i=1}^{p} \Pi\left(W_{i}\right)\right) \\
\leqq & 3 \frac{|z|}{1-|z|}\left(\Pi(W)+\Pi\left(\theta^{\prime \prime}\right)\right)
\end{aligned}
$$

for $|z|$ small enough using the remark following the proof of Theorem 5 . We observe also that

$$
\sum_{i=1}^{n}\left|V\left(\theta, \theta_{i}\right)\right| \text { with } 0 \neq \theta_{i} \leqq \theta^{\prime \prime}, \quad \theta_{i} \neq \theta_{j} \forall i, j
$$

is certainly smaller than

$$
\sum_{0 \neq \theta_{i} \leqq \theta^{\prime \prime}}\left|V\left(\theta, \theta_{i}\right)\right| \leqq 3 \frac{|z|}{1-|z|}\left(\Pi(W)+\Pi\left(\theta^{\prime \prime}\right)\right)
$$

for $|z|$ small enough. Finally we notice that $0 \neq \theta_{i} \leqq \theta^{\prime \prime}$,

$$
\sum_{i=1}^{n} \theta_{i} \geqq \theta^{\prime \prime}
$$

imply

$$
\sum_{i=1}^{n} \delta\left(W+\theta_{i}\right)+|p(W)| \geqq \delta\left(W+\theta^{\prime \prime}\right) .
$$

We get using (A-6), (A-7), (A-8) and $|p(W)| \leqq \Pi(W)$ the upper bound $(R>1)$

$$
\begin{aligned}
\sum_{\substack{\theta^{\prime \prime} \\
\theta+\theta^{\prime \prime} \text { adm. }}}\left|K\left(\theta, \theta^{\prime \prime}\right)\right| R^{-\Pi\left(\theta^{\prime \prime}\right)}-1 \leqq & \sum_{\substack{0 \neq \theta^{\prime \prime} \\
\theta+\theta^{\prime \prime}}} R^{-\Pi\left(\theta^{\prime \prime}\right)} R^{-\delta\left(W+\theta^{\prime \prime}\right)} R^{\Pi(W)} \\
& \cdot \exp \left(\frac{6|z|}{1-|z|}\left(\Pi(W)+\Pi\left(\theta^{\prime \prime}\right)\right)\right)
\end{aligned}
$$


The sum is equal to

$$
\begin{aligned}
& R^{2 \Pi(W)} \sum_{\substack{0 \neq \theta^{\prime \prime} \\
\theta+\theta^{\prime \prime} \text { adm. }}} R^{-\delta\left(W+\theta^{\prime \prime}\right)}\left[R^{-1} \exp \frac{6|z|}{1-|z|}\right]^{\Pi\left(\theta^{\prime \prime}\right)+\Pi(W)} \\
& \leqq R^{2 \Pi(W)} \sum_{\substack{W \leqq \theta^{\prime \prime} \\
\theta^{\prime \prime} \text { adm. } \theta^{\prime \prime} \neq W}} R^{-\delta\left(\theta^{\prime \prime}\right)}\left[R^{-1} \exp \frac{6|z|}{1-|z|}\right]^{\Pi\left(\theta^{\prime \prime}\right)} .
\end{aligned}
$$

For fixed $|z|$ and $R$ large enough the last sum is convergent (see Proof of Theorem 1) and is bounded by

$$
K_{R} \Pi(W)\left[R^{-1} \exp \frac{6|z|}{1-|z|}\right]^{\Pi(W)}
$$

where $K_{R}$ is a constant depending on $R$ and such that $K_{R} \rightarrow 0$ as $R \rightarrow \infty$. $\left(\theta^{\prime \prime} \neq W\right.$ implies $\delta\left(\theta^{\prime \prime}\right)>0$ ). Therefore

$$
\sum_{\substack{\theta^{\prime \prime}: \\ \theta+\theta^{\prime \prime} \text { adm. }}}\left|K\left(\theta, \theta^{\prime \prime}\right)\right| R^{-\Pi\left(\theta^{\prime \prime}\right)} \leqq 1+K_{R} \Pi(W)\left[R \exp \frac{|z|}{1-|z|}\right]^{\Pi(W)}
$$

and we have the following upper bound for the square brackets in (A-5)

$$
\alpha(|z|, R)^{\Pi(W)}\left(1+K_{R} \Pi(W) \beta(|z|, R)^{\Pi(W)}\right)
$$

with

$$
\begin{aligned}
& \alpha(|z|, R)=|z| R \exp \left(\frac{3|z|}{1-|z|}+C\right) \\
& \beta(|z|, R)=R \exp \frac{6|z|}{1-|z|} .
\end{aligned}
$$

For a given $R$ we can choose $r_{2}(R)$ so that if $|z| \leqq r_{2}(R)$ we have

$$
I_{m+1}^{R} \leqq|z| I_{m}^{R}
$$

since $\Pi(W) \geqq 4$.

Let us finish now the proof of the theorem.

$$
\begin{aligned}
I_{1}^{R} & =\sup _{W}|\varphi(W)| R^{\Pi(W)} \leqq \sup _{W}(R|z|)^{\Pi(W)} \exp \left|U_{1}(W)\right| \\
& \leqq \sup _{W}\left(R|z| \exp \frac{3|z|}{1-|z|}\right)^{\Pi(W)} \leqq|z|, \quad|z|<r_{2} .
\end{aligned}
$$

Therefore $I_{m}^{R} \leqq|z|^{m},|z|<r_{2}(R)$ and since $I_{m}^{R}$ is monotone in $R$ we can find for any $R$ an $r(R)$ (monotone decreasing in $R$ ) such that $r(R) \rightarrow 0$ as $R \rightarrow \infty$ and

$$
I_{m}^{R} \leqq|z|^{m} \text { if }|z|<r(R) .
$$

Using the algebraic formalism one shows that

$$
\rho_{L}(\theta)=\sum_{\theta^{\prime} \subset A_{L}} \Delta_{\theta}\left(\theta^{\prime}\right) \text {. }
$$


Using (A-4) and the above lemma we have

$$
\begin{aligned}
\left|\rho_{L}(\theta)\right| & \leqq R^{-\Pi(\theta)} \sum_{m \geqq N(\theta)} \sum_{N\left(\theta+\theta^{\prime}\right)=m}\left|\Delta_{\theta}\left(\theta^{\prime}\right)\right| R^{\Pi(\theta)} \\
& \leqq R^{-\Pi(\theta)} \frac{|z|^{N(\theta)}}{1-|z|} \\
\sum_{\text {Int } \theta \ni x_{0}}\left|\varphi^{T}(\theta)\right| & =\sum_{\text {Int } W \ni x_{0}} \sum_{\theta}\left|\varphi^{T}(W+\theta)\right| \\
& \leqq \sum_{\text {Int } W \ni x_{0}} R^{-\Pi(W)} \sum_{m=1}^{\infty} I_{m}^{R} \leqq C_{R} \frac{|z|}{1-|z|}
\end{aligned}
$$

where $C_{R} \rightarrow 0$ as $R \rightarrow \infty$. We choose $r(R)$ so that $C_{R}<1$.

The functions $\Delta_{\theta}\left(\theta^{\prime}\right)$ are analytic in $z$ because they are polynomials in $\varphi_{L}(\theta)$ which are themselves analytic functions (see Section 4). From this we get the analyticity of $\rho_{L}(\theta)$ since $\sum_{\theta^{\prime}} \Delta_{\theta}\left(\theta^{\prime}\right)$ is a uniformly bounded absolutely convergent series by (A-9).

\section{Appendix 3}

\section{Widom Rowlinson Models}

For simplicity we consider only the model which we treated in detail in [3] and [4]. We refer the reader to those papers for notations and definitions. We consider a pure $A$ boundary condition in a volume $\Lambda$. Let $\left(S_{i}, i \in \Lambda\right)$ be a configuration in $\Lambda$ such that the set $\gamma$ of empty sites in this configuration is connected. We define Int $\gamma$ as the set of sites where we have a $B$ particle and Ext $\gamma$ as the set of sites where we have an $A$ particle.

Definition. A contour is the couple $(\gamma, \operatorname{Int} \gamma)$

For each contour we can define

$$
S_{i}(\gamma)=\left\{\begin{aligned}
+1 & \text { if } i \in \operatorname{Ext} \gamma \\
0 & \text { if } i \in \gamma \\
-1 & \text { if } i \in \operatorname{Int} \gamma
\end{aligned}\right.
$$

It is not difficult to see that any configuration in $\Lambda$ with $A-b . c$. can be decomposed uniquely in contours so that the value of $S_{i}$ in the configuration is given by

$$
\prod_{\gamma} S_{i}(\gamma)
$$

the product being over all contours of the configuration. Therefore we can prove Theorem 3 and since the analysis of the interface is the same as before we also have Theorem 1 . However the existence of the limits for real activity in this theorem does not follow by correlation inequalities but we may use either the results of [4] that prove the existence of these limits or use the methods of this paper. 
Acknowledgements. It is a pleasure to thank J. Slawny for very valuable discussions and H. Kunz and B. Souillard for communicating to us Theorem 3 prior to publication.

\section{References}

1. Dobrushin, R. L. : Theory Probab. Its Appl. 17, 582 (1972)

2. Dobrushin, R. L. : Theory Probab. Its Appl. 18, 253 (1973)

3. Bricmont, J., Lebowitz, J. L., Pfister, C. E., Oliveri, E. : Commun. Math. Phys. 66, 1-20 (1979)

4. Bricmont, J., Lebowitz, J. L., Pfister, C. E. : Commun. Math. Phys. 66, 21-36 (1979)

5. Gallavotti, G. : Commun. Math. Phys. 27, 103 (1972)

6. Minlos, R. A., Sinai, J. : Trans. Moscow Math. Soc. 17, 237 (1967); Math. USSR-Sbornik 2, 355 (1967); Trans. Moscow Math. Soc. 19, 121 (1968)

7. Gallavotti, G., Martin-Löf, A., Miracle-Sole, S. : In: Statistical mechanics and mathematical problems, Battelle Rencontres 1971. Lecture notes in physics, Vol. 20. Berlin, Heidelberg, New York: Springer 1973

8. Kunz, H., Souillard, B. : Private communication, to appear

9. Gruber, C., Hintermann, A., Messager, A., Miracle-Sole, S. : Commun. Math. Phys. 56, 147 (1977)

10. Messager, A., Miracle-Sole, S. : J. Stat. Phys. 17, 245 (1977)

11. Holsztynski, W., Slawny, J. : Commun. Math. Phys. 66, 147-166 (1979)

12. Slawny, J. : in preparation

Communicated by A. Jaffe

Received May 10, 1979 
